\title{
Utilidad preventiva del constructo "trastorno mental grave" en el ámbito sociosanitario
}

\author{
Preventive usefulness of the concept "serious mental disorder" in social-sanitary area \\ José Carlos Mingote Adán \\ UVOPSE-PAIPSE. Unidad de Valoración y Orientación al profesional \\ sanitario enfermo. Comunidad de Madrid. Madrid. España
}

Pablo del Pino Cuadrado

UVOPSE-PAIPSE. Unidad de Valoración y Orientación al profesional sanitario enfermo. Comunidad de Madrid. Madrid. España

Macarena Gálvez Herrer

UVOPSE-PAIPSE. Unidad de Valoración y Orientación al profesional sanitario enfermo. Comunidad de Madrid. Madrid. España

\section{M. ${ }^{a}$ Dolores Gutiérrez García \\ UVOPSE-PAIPSE. Unidad de Valoración y Orientación al profesional sanitario enfermo. Comunidad de Madrid. Madrid. España}

\section{Raquel Sánchez Alaejos}

UVOPSE-PAIPSE. Unidad de Valoración y Orientación al profesional sanitario enfermo. Comunidad de Madrid. Madrid. España

Recibido: 03-12-10

Aceptado: 07-12-10

\section{Correspondencia:}

José Carlos Mingote Adán.

UVOPSE-PAIPSE

Pabellón n.` 8 Ciudad Universitaria

28040 Madrid. España.

Tfno: 913303926

Fax: 913303903

E-mail: jmingote.hdoc@salud.madrid.org

\section{Agradecimientos}

En la elaboración de este documento se han tenido en cuenta los Protocolos Asistenciales y de Funcionamiento del Programa de Atención Integral al Médico Enfermo (PAIME) del Consell de Collegis de Metges de Catalunya, así como las recomendaciones de la Comisión de Ayuda y Prevención al Médico Enfermo (CAYPAM) del Ilustre Colegio de Médicos de Madrid y los Códigos Deontológicos de los Colegios Profesionales de Enfermería y de Médicos. Nuestro más sincero agradecimiento a todos ellos.

Agradecemos además la revisión de su contenido a: D. Francisco Javier Sánchez Caro (Responsable del Área de Bioética y Derecho Sanitario, Dirección General de Atención al Paciente, Consejería de Sanidad), Dr. Andrés Santiago Sáez (Jefe de Servicio de Medicina Legal del Hospital Clínico San Carlos), Dr. César Borobia (Profesor Titular de Medicina Legal, Ftad. Medicina U.C.M.), Dr. Alfredo Calcedo Ordóñez (Catedrático de Psiquiatría, Co-Director Master Psiquiatría Legal, U.C.M), Dr. Jerónimo Maqueda (Director de la Escuela Nacional de Medicina del Trabajo) y Dra. Rosana Cortés (Jefa de Estudios de la Nacional de Medicina del Trabajo) y al Comité Técnico Asesor de la UVOPSE-PAIPSE.

"El diagnóstico es pronóstico"

(Godwin y Guze, 1974) 


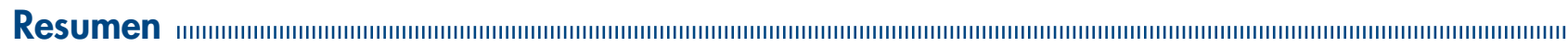

Introducción: El constructo "trastorno mental grave" (TMG) puede ser muy útil en la práctica clínica y no sólo en el ámbito de la gestión de los recursos, al permitir diseñar intervenciones preventivas, diagnósticas y terapéuticas efectivas, especialmente en el sector servicios y en profesiones de alta fiabilidad y seguridad (sanidad, defensa, transporte, etc.) en las que los errores suelen tener consecuencias directas adversas para la vida de las personas y los que se debe garantizar la máxima calidad y la seguridad de los usuarios.

Objetivos: Revisar los principales aspectos a considerar en la elaboración de protocolos de intervención laboral ante trastornos mentales graves.

Método: Revisión de la bibliografía disponible.

Conclusiones: Se proponen herramientas y procedimientos de utilidad para afrontar los casos clínicos graves en el ámbito laboral sanitario, mejorar la calidad asistencial y contribuir a garantizar la seguridad de los usuarios y de los sanitarios enfermos.

Med Segur Trab (Internet) 2010; 56 (221): 306-322

Palabras claves: Trastorno mental grave. Criterios diagnósticos. Prevención. Intervención.

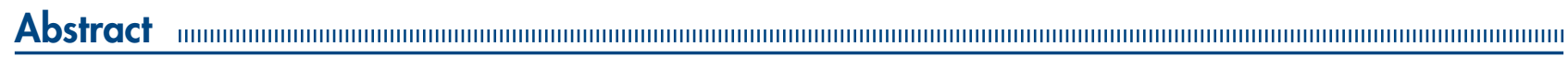

Introduction: The concept of "serious mental disorder" (SMD) can be very useful in clinical medical practice, not only in the management resources area, allowing to design preventive, diagnostic and therapeutic effective interventions, but also in service area and in high reliability and security professions, such as health, defence, transport and so on. In these cases mistakes use to have direct and adverse consequences for people's life that is way a maximum quality and safety must be guaranteed for the users.

Objective: To check the principal aspects to consider in the production of intervention protocols for mental serious disorders in the workplace.

Method: Review of the available bibliography.

Conclusions: This article proposes tools and procedures to deal with serious clinical cases in health professional field in order to improve the attendance quality, contributing to the safety of the health workers and users.

Med Segur Trab (Internet) 2010; 56 (221): 306-322

Key words: Serious mental health. Diagnostic criteria. Prevention. Intervention. 


\section{INTRODUCCIÓN}

Dada la actual crisis nosográfica, conviene recordar las advertencias de Jaspers ${ }^{1}$ : “...es posible rechazar todos los esfuerzos realizados. Se puede decir: No hay ninguna unidad nosológica, se corrió tras un fantasma". Esta crisis nosográfica que vive la Psiquiatría como disciplina médica es reflejo de la complejidad constitutiva del ser humano y de la evidencia científica actual, que requiere el empleo de modelos teóricos integradores e instrumentos válidos de medida de la enfermedad que puede padecer una persona en un momento determinado. Casi 100 años más tarde, es preciso reconocer que ha mejorado mucho la fiabilidad de los diagnósticos psiquiátricos cuando se emplean las herramientas apropiadas, para los diferentes síndromes clínicos. La mayor parte de ellos se definen por su principal característica sintomática, que ninguna es específica de un trastorno mental concreto, pero aún no se ha demostrado que sean entidades discretas o categorías nosológicas válidas separadas por límites naturales o verdaderas discontinuidades explicables por la existencia de un sustrato biológico diferente para cada una de ellas.

Actualmente, se propone la clasificación dimensional de los trastornos mentales, separados por límites arbitrarios decididos por consenso de expertos ${ }^{2}$. Otra cosa diferente es la utilidad clínica de un diagnóstico formal concreto, en la medida en que proporciona información valiosa de tipo pronóstico acerca de la respuesta al tratamiento. La utilidad clínica del constructo "trastorno mental grave" (en adelante, TMG) dependerá de su validez predictiva para anticipar la evolución del enfermo y la administración de los tratamientos más efectivos. Pero quedarnos ahí supondría una visión reduccionista, su consideración también puede contribuir a mejorar la seguridad de los usuarios y de los sanitarios, entendida ésta desde una perspectiva sistémica (que incluye la consideración de los medios materiales, los procedimientos técnicos y organizativos, así como la selección y el cuidado de los operadores especialmente cualificados por su resistencia al estrés y la toma de de decisiones de alto rendimiento), sobre todo en situaciones de urgencia y de riesgo vital.

La protocolización de la atención a la Salud Mental ha sido abordada recientemente como una herramienta eficaz orientada a la mejora de la calidad y seguridad de la asistencia al usuario, así como a facilitar la práctica del profesional sanitario que de él se ocupa. El objetivo del presente trabajo es analizar el concepto de TMG en relación con el posible deterioro funcional a partir del cual pueda suponer un riesgo para sí mismo o para terceros durante la actividad laboral. Se revisarán los principales aspectos a considerar en la elaboración de protocolos de intervención laboral ante dichos trastornos (principalmente en personal sanitario) y la prevención de sus consecuencias en el medio laboral, concretamente se analizarán aspectos a considerar y definir en un posible protocolo y procedimientos de actuación recomendados.

\section{ASPECTOS A DEFINIR}

\section{Capacidad y competencia profesional}

Una primera cuestión a plantearse ante un posible TMG en el lugar de trabajo es su capacidad y competencia profesional para el desempeño seguro de sus funciones. En nuestro ámbito legal, por el mero hecho del nacimiento en las condiciones establecidas en el Código Civil (artículos 29 y 30) se es persona, esto es, se tiene personalidad y por el mero hecho de ser persona y tener personalidad se es capaz, siendo importante distinguir entre capacidad jurídica y capacidad de obrar:

- La capacidad jurídica: Es la abstracta posibilidad de recibir los efectos del ordenamiento jurídico.

- Capacidad de obrar: aptitud para ejercer personalmente un derecho y el cumplimiento de una obligación, esto es, la aptitud legal para ser sujeto de derechos y obligaciones. 
La competencia: Presupone siempre la capacidad de obrar (posibilidad de realizar actos con eficacia jurídica) y añade otros elementos como la preparación profesional o académica adecuada, la idoneidad para dicha profesión en el sentido de que se puede realizar la tarea profesional concreta y habilidades mayores o menores según la circunstancia y técnica concreta.

La competencia médica ha sido definida como ${ }^{3}$ : "La capacidad de utilizar de manera habitual y razonable la comunicación, los conocimientos, las habilidades técnicas, la capacidad para la toma de decisiones basadas en la evidencia, las emociones, los valores, y la reflexión en beneficio del individuo y de la comunidad que se atiende". Incluye los siguientes aspectos o dimensiones básicas ${ }^{4}$ :

1. Cognitivos: Adquirir y utilizar conocimientos.

2. Integrados: Sintetizar datos, razonar y juzgar.

3. Relacionales: Comunicarse con pacientes y colegas.

4. Afectivos/éticos: Demostrar paciencia, voluntad y autoconocimiento.

Además de las capacidades adquiridas a través de la formación continua y la experiencia laboral, la competencia profesional también se define por las condiciones de salud del personal que facilitan o interfieren en su puesta en práctica, tal y como señalan los Códigos Deontológicos 5: "La enfermera mantendrá un nivel de salud personal que no comprometa su capacidad para dispensar cuidados".

Según el Plan de Bolonia para los Estudios de Grado de Medicina ${ }^{6}$, el médico necesitará contar con las suficientes competencias para su desarrollo profesional tales como: autocrítica y auto-evaluación, aprendizaje autónomo y cuidado personal; incluyendo ser capaz de atender a su estilo de vida, requerir ayuda y consejo en situaciones difíciles, reconocer los peligros de automedicación y abuso de sustancias, conocer la influencia y prevención de factores relacionados con su trabajo para la salud propia y ajena.

Según el Documento Sitges ${ }^{7}$, la capacidad de autonomía personal se puede encontrar limitada tanto por límites "naturales", como "legales", entre los que destacan los derechos de los demás y el perjuicio inaceptable de tercero, que condicionan y limitan el marco de las decisiones individuales. Es decir, se deberá tener en cuenta tanto las condiciones necesarias para la toma de decisiones, como la trascendencia individual y/o social de la misma. La toma de decisiones depende especialmente de la disponibilidad de los recursos cognitivos, emocionales y conductuales de cada persona en un momento dado.

En el caso de los sanitarios, con responsabilidad directa o indirecta sobre la vida de terceros, la seguridad (peligrosidad) mencionada anteriormente, constituye un elemento fundamental de su actividad cotidiana. A su vez, los procesos de conflicto interpersonal en el trabajo (con compañeros y/o pacientes) son posibles indicadores de la existencia de un trastorno mental y/o de problemas organizativos que repercuten en la salud de los trabajadores.

\section{Gravedad de los trastornos y factores de riesgo a controlar}

El término gravedad clínica se aplica a enfermedades que ponen en peligro la vida de las personas. Así puede ocurrir por ejemplo, en el caso de una depresión psicótica con intensa impulsión suicida, por la cualidad, intensidad y repercusión de algunos síntomas en la vida del paciente y de otras personas. Los síntomas de los diferentes trastornos mentales se han dividido entre los que producen sufrimiento y dolor, y los que causan pérdida funcional o discapacidad, frente al malestar normal de la vida. El término psicótico es sinónimo de deterioro grave del funcionamiento personal y social, caracterizado por la incapacidad para desarrollar con normalidad los roles familiares y laborales por alteración grave del contacto con la realidad. 
La gravedad del trastorno mental actual debe incluir tanto la sintomatología psíquica, como los problemas psicosociales asociados, la sintomatología somática y otros problemas tales como son los conflictos interpersonales en el trabajo y los problemas de adaptación al medio sociolaboral. En general, existe una relación dosis-respuesta entre la gravedad de la enfermedad mental y el grado de incapacidad laboral asociado ${ }^{8,9}$.

Una enfermedad mental grave es un trastorno con deterioro funcional ${ }^{10}$, y se define de la siguiente manera:

1. Trastorno: Cualquier trastorno DSM diagnosticado durante un período de 12 meses, excluyendo los códigos $\mathrm{V}$ (afecciones no atribuibles a un trastorno mental que son objeto de atención clínica o tratamiento, como los problemas académicos o la simulación), los trastornos por consumo de sustancias (que en el próximo DSM-V se ha propuesto llamarlo "Adicciones y Trastornos Relacionados", incluyendo juego patológico y adicción a Internet), y los trastornos del desarrollo.

2. Deterioro: Interferencia considerable en al menos una de las principales actividades de la vida que incluyen no sólo las actividades básicas de la vida cotidiana como comer o bañarse, sino también "las habilidades instrumentales (p. ej., mantener un hogar, manejar dinero, desenvolverse en sociedad, tomar la medicación prescrita), y el funcionamiento en el contexto social, familiar y laboral/educativo".

No existe una definición consensuada sobre el constructo de TMG, éste puede variar según que el objetivo prioritario sea la planificación y gestión sanitaria, la investigación, la intervención clínica, etc., y son numerosas las posibles definiciones que se pueden encontrar al respecto en la bibliografía científica. Una revisión de las mismas ${ }^{11}$ indica como elementos comunes la necesidad de prestar atención a tres dimensiones:

- Diagnóstico: Incluyendo, de forma prioritaria, la psicosis y trastornos crónicos.

- Duración: Variable según las diferentes definiciones desde 1 a 5 años desde el inicio del trastorno.

- Discapacidad: Operativizada en función del desempeño de roles sociales, familiares, laborales y de autocuidado. Según estos autores, la efectividad de los Servicios Sanitarios depende tanto de la calidad de la tecnología instrumental como de la tecnología humana (el factor humano), siendo tan importante la competencia científico-técnica personal, como los factores derivados de la actitud y de la personalidad.

Otros autores ${ }^{12}$ proponen el sistema definido por las dimensiones Safety, Informal, Diagnosis, Disability and Duration (SIDD) como elemento de definición de TMG:

1. Seguridad (peligrosidad). Hacia sí mismo o hacia terceros.

2. Apoyo (soporte): Social e institucional.

3. Diagnóstico: Incluyendo enfermedad psicótica, demencia, enfermedad neurótica grave, trastorno de la personalidad y trastorno del desarrollo.

4. Discapacidad: Con habilidades disminuidas para funcionar eficazmente en la comunidad (trabajo, familia, relaciones interpersonales).

5. Duración: Persistencia de cualquiera de las dimensiones mencionadas (6 meses-2 años). clínicos:

Según consenso genera $1^{13}$ se entiende por TMG el que cumple los siguientes criterios

a) Trastorno mental psicótico (esquizofrenia, paranoia, depresión psicótica, etc.) o adictivo, en especial si es crónico y/o recurrente, y trastornos graves de la personalidad (sobre todo los del Cluster A y B del DSM).

b) Trastorno que ha requerido un tratamiento prolongado, igual o superior a dos años.

Utilidad preventiva del constructo "trastorno mental grave" en el ámbito sociosanitario 
c) Trastorno que se asocia a un descenso en el rendimiento funcional y muestra discapacidad en sus actividades diarias (como en el trabajo y en sus relaciones interpersonales) con una puntuación igual o inferior a 50 en la Escala de evaluación de la actividad global (DSM-IV- TR).

Los parámetros de gravedad en adultos a considerar serán ${ }^{14}$ :

a) La gravedad diagnóstica (psicosis, depresión mayor, trastorno neurótico severo, crisis adaptativa severa, alcoholismo y adicciones severas, trastornos alimentarios graves) combinada o no con situaciones o grupos de riesgo.

b) Comorbilidad de distintas patologías psíquicas o físicas.

c) Grado de discapacidad producido (en relación con autocuidado y salud, afrontamiento personal y social, autonomía personal y social y tratamiento).

d) Repercusión del trastorno sobre el medio familiar y social (valorando el riesgo de heteropeligrosidad).

e) Autopeligrosidad (riesgo autolítico).

f) Precariedad o agravantes de la red social (soporte social y factores que dificulten la contención y/o obstaculicen el tratamiento).

g) Características de la demanda (voluntaria o involuntaria, nivel de sufrimiento y motivación).

h) Tiempo de evolución del trastorno (agudo o crónico).

i) Complejidad del manejo terapéutico (prestaciones necesarias, trabajo en red y necesidad de coordinación entre diferentes servicios).

j) Aparición de los síntomas en etapas de especial relevancia evolutiva (por ejemplo al inicio de la actividad laboral).

Respecto a los trastornos adictivos, que por definición son también trastornos crónicorecurrentes (caracterizados por el consumo compulsivo, la pérdida de control y la producción de estados emocionales negativos asociado todo ello a un elevado riesgo de estados emocionales negativos, disfunciones cognitivas y conductuales que suponen un alto riesgo de mala praxis), se hace necesario, no solo realizar un tratamiento completo sino controlar el cumplimiento terapéutico y prevenir las posibles recaídas a largo plazo.

Para analizar la gravedad del trastorno relacionado con el abuso de sustancias adictivas, se valorarán los siguientes criterios: cantidad, frecuencia y antigüedad del uso de la sustancia, así como las percepciones del paciente, déficit de control y las repercusiones psicopatológicas, afectivas, cognitivas y conductuales. Para ello se podrán utilizar cuestionarios autoadministrados, pruebas de laboratorio y análisis de sustancias en sangre, orina y cabello (art. 785 Ley de Enjuiciamiento Criminal). Estas pruebas sirven tanto para objetivar el consumo de una sustancia, gravedad del trastorno, cumplimiento terapéutico y grado de abstinencia (siempre salvaguardando la confidencialidad de los resultados).

En resumen, según criterios de gravedad y deterioro funcional expuestos, serán de especial atención los siguientes casos ${ }^{15}$ :

1. El trastorno esquizo-afectivo, maniaco-depresivo, autismo, formas graves de depresión mayor, trastorno de angustia o el trastorno obsesivo-compulsivo, puesto que si no se tratan suelen provocar un importante deterioro.

2. En algún momento, durante los últimos 12 meses, el trastorno interfirió considerablemente con la capacidad laboral, causó grandes dificultades interpersonales, o estuvo asociado a un plan o tentativa suicida.

3. En algún momento, a lo largo de los últimos 12 meses, se cumplieron los criterios de deterioro funcional sin que el tratamiento u otros servicios de apoyo fuesen efectivos. 
El concepto de discapacidad deberá valorarse en cada caso individual como un disbalance persistente de la interacción entre "la condición de salud" del individuo enfermo y las demandas adaptativas del medio sociolaboral específico ${ }^{16}$. Además, consideramos importante señalar que en caso de TMG se pueden presentar las siguientes características:

1. Carencia de capacidad de adaptación a la realidad y en especial a situaciones estresantes.

2. Inflexibilidad desadaptativa rígida (compulsión de repetición) o claudicación funcional adaptativa grave.

3. Importantes dificultades para aprender de la experiencia y desarrollar nuevas estrategias adaptativas eficaces.

4. Malestar clínicamente significativo.

5. Importante conflictividad interpersonal y/o aislamiento social.

Los déficit cognitivos en los TMG son indicadores diagnósticos de gravedad en varios trastornos mentales que comparten algunos déficit neuropsicológicos característicos, así como biomarcadores neurofisiológicos mediadores cada vez mejor conocidos, como ocurre con los niveles plasmáticos elevados de cortisol y los déficit de memoria que se aprecian en personas con esquizofrenia, depresión, trastorno de estrés postraumático crónico, síndrome de Cushing y demencia. Habitualmente se aprecian déficit en atención sostenida, en el procesamiento de información compleja y en las funciones ejecutivas. Estos déficits neuropsicológicos no difieren significativamente entre pacientes con esquizofrenia, trastorno esquizoafectivo y trastorno bipolar psicótico, sino que parecen extenderse a lo largo de todo el espectro psicótico, con diferencias cuantitativas entre unos y otros trastornos ${ }^{17,18}$. Estos déficit neuropsicológicos parecen ser marcadores de rasgo de los trastornos mentales graves, dependientes de factores genéticos y epigenéticos precoces, más que un déficit específico de un trastorno mental. En todos los casos la presencia de disfunción neuropsicológica tiene una importante significación pronostica, tanto en cuanto al curso evolutivo clínico como al funcionamiento sociolaboral de personas con un amplio rango de trastornos mentales graves ${ }^{19}$.

Los déficits neuropsicológicos señalados, limitan la capacidad o determinan incapacidad para la introspección y la autorregulación, la inteligencia emocional y social, la toma de decisiones y el procesamiento de información compleja, según un juicio de realidad sano.

Algunos estudios epidemiológicos ${ }^{20}$ encuentran que la prevalencia anual de trastornos mentales moderados o graves en población general trabajadora está en torno al 14\%, sea por la agravación de cualquier trastorno mental previo en algún momento de su evolución o por la cronificación y la complicación del mismo con trastornos adictivos y con enfermedades médicas graves, con los que presentan una elevada comorbilidad habitual.

Para hacer operativos los criterios de gravedad y deterioro funcional se recomiendan los siguientes cuestionarios de evaluación que pueden ser incluidos en protocolos de prevención y/o procedimientos de actuación:

- Escala de Evaluación del Insight (SAI) ${ }^{21}$.

- Escala de Gravedad de la Enfermedad Psiquiátrica para pacientes ambulatorios $\left(\right.$ Gep-P) ${ }^{22}$.

- Instrumento Mac Arthur para la Evaluación de la capacidad sobre el tratamiento $\left(\right.$ Mac Cat-T) ${ }^{23}$. 


\section{PROCEDIMIENTOS DE ACTUACIÓN}

\section{Evaluación y definición de la población diana}

El primer paso de cualquier procedimiento de actuación debe ser la evaluación y definición de la población diana del mismo. Se debe evaluar la gravedad de síntomas tales como delirios y alucinaciones, la ausencia de insight, la pérdida de contacto con la realidad y el deterioro del funcionamiento mental. Además, es preciso evaluar el sufrimiento subjetivo, la evolución temporal (cronicidad y recurrencia), la pérdida de función o discapacidad que producen los síntomas, así como el riesgo predecible para la persona enferma y para sus semejantes. Respecto de la función sociolaboral es preciso evaluar la extensión de la alteración en una o más áreas importantes de actividad, como son el trabajo, las relaciones interpersonales, el cuidado de uno mismo y/o de los demás, etc. ${ }^{24}$

La evaluación psicológica debe incluir:

1. Realización de una historia clínica completa con la evaluación del estado mental actual y los antecedentes psicológicos personales.

2. Evaluación neuropsicológica global.

3. Evaluación de personalidad que incluya motivación y orientación laboral, capacidad para tomar decisiones en situaciones complejas, habilidades sociales y de afrontamiento del estrés por la disponibilidad de los recursos cognitivos, afectivos y conductuales adecuados.

Según la bibliografía revisada en el apartado anterior, se recomienda considerar como población diana de los procedimientos de actuación, los siguientes TMG:

A) Pacientes con los siguientes trastornos mentales y características asociadas:

- Trastorno psicótico endógeno o inducido por drogas.

- Trastorno delirante crónico endógeno o inducido por drogas.

- Trastorno bipolar tipos I, II y III si existe comorbilidad con trastorno adictivo.

- Demencia y deterioro cognitivo de causa psico-orgánica (trastorno mental severo, drogas, etc.)

- Paciente con diagnóstico de trastorno grave de la personalidad, si existe comorbilidad con consumo de drogas (de forma prioritaria en caso de trastorno de tipo límite, antisocial o paranoide).

- Trastorno disruptivo de conducta.

- Trastorno mental grave que precise ingreso psiquiátrico.

B) Pacientes con trastornos adictivos y con las siguientes características:

- Trastorno adictivo y ejercicio de especialidad de riesgo (como anestesia, cirugía, ginecología, urgencias, intensivos, farmacología clínica, farmacia hospitalaria y demás servicios intervencionistas).

- Presencia de patología dual.

- Presencia de consumo durante la práctica profesional y/o en el lugar de trabajo (detectado en anamnesia).

- Fracaso previo en al menos dos tratamientos frente a la adicción.

- Policonsumo.

Una vez establecida la existencia de un TMG se debe evaluar la posible existencia de una restricción funcional significativa secundaria al mismo, en las actividades de la vida diaria, en el trabajo o en el medio familiar, así como la existencia de deficiencias de concentración, persistencia, ritmo de trabajo, etc. que además puede llegar a generarle un estrés laboral que puede descompensarle, además de poder implicar riesgos para terceros. Para ello es preciso realizar el estudio protocolizado de su capacidad funcional en: comprensión, memoria, 
atención-concentración, interacción social y disponibilidad de recursos adaptativos adecuados a las demandas del rol profesional específico. Carrasco y Maza ${ }^{25}$ recomiendan además, utilizar los criterios del DSM-IV-TR del Eje V y las Escalas de Actividad (Global, Social y Laboral y Relacional) para definir la existencia de deterioro funcional.

\section{Criterios de detección del riesgo para sí mismo o para terceros}

Un objetivo prioritario de cualquier procedimiento de actuación ante el TMG en el entorno laboral debe ser la detección del riesgo para sí mismo y/o para terceros. Esto puede ser algo de difícil definición, entre otras cosas porque la mayor parte de las personas con TMG no tienen conciencia de enfermedad mental ni demandan asistencia sanitaria de forma voluntaria, sino que suelen hacerlo inducidos por familiares y allegados, o bien de forma involuntaria ${ }^{26}$.

Durante la evaluación del paciente se recomienda valorar la existencia de riesgo mediante:

- La confirmación del mismo por el propio paciente a lo largo de la entrevista de apertura de historia y/o la entrevista clínica.

- Información al respecto por parte del Servicio de Prevención de Riesgos Laborales que corresponde al paciente.

- Quejas y/o reclamaciones de pacientes sobre ese profesional recogidas por escrito, que hagan presuponer el riesgo.

- Manifestaciones en este sentido, reiteradas y concretas, de compañeros y/o supervisores.

- Manifestaciones en este sentido por parte de familiares o acompañantes.

- Conocimiento del terapeuta responsable del caso al realizar la evaluación y abordaje del mismo.

Se tendrán en consideración los siguientes elementos:

- Historia psiquiátrica y laboral previa.

- Tipo de diagnóstico/s.

- Gravedad clínica. Identificando la presencia de:

- Pensamientos de auto / hetero-agresión.

- Ideación autolítica intensa.

- Delirios o alucinaciones francas.

- Cuadro de agitación o pérdida del autocontrol.

- Patología orgánica grave asociada.

- Situación de consumo y/o abstinencia de drogas que comporte un peligro para la praxis.

- Especialidad de riesgo (médicos y enfermeros de urgencias, de cuidados intensivos, cirujanos, anestesistas, y/o otros puestos de elevada responsabilidad respecto a terceros).

- Condición de "trabajador especialmente sensible a determinados riesgos" informada por su correspondiente Servicio de Prevención de Riesgos Laborales (en función del Art. 25 de la Ley de Prevención de Riesgos Laborales).

- Condiciones de trabajo de elevado riesgo psicosocial.

Por otro lado, habitualmente serán las personas próximas al trabajador las que con mayor probabilidad detecten las complicaciones asociadas al posible TMG. Las señales de alarma a detectar y el personal implicado en ello serán, principalmente, los siguientes:

- Comportamiento alterado con patrones desadaptativos de conducta e incumplimiento manifiesto de las demandas propias del rol profesional como: Excesiva e inadecuada irritabilidad, apatía, inhibición y falta de responsabilidad en 
el cumplimiento de las tareas propias del rol profesional, aislamiento, problemas de comunicación y/o frecuentes conflictos interpersonales, convenientemente objetivados por varios observadores externos.

- Hiperreactividad al estrés cotidiano con baja tolerancia a situaciones estresantes comunes: como cambios en rutinas laborales, situaciones de incertidumbre y conflictos inherentes a la actividad sanitaria.

- Incumplimiento de los hábitos básicos de autocuidado: hábitos de salud y aspecto externo.

- Padecimiento de enfermedades crónicas (como las neurológicas) que impliquen deterioro de la capacidad funcional, necesidad de asumir el rol de enfermo y ausencia de conciencia de enfermedad.

- Situaciones estresantes específicas graves como la muerte de un familiar próximo, separación de la pareja, situaciones económicas desfavorables.

Personal implicado en la detección:

- El propio trabajador: En este sentido, parece que lo expuesto para los profesionales médicos en su Código de Ética y Deontología Médica (Art. 19) ${ }^{27}$ puede ser orientativo para la actuación de todos los profesionales sanitarios:

a) El médico debe abstenerse de actuaciones que sobrepasen su capacidad. En tal caso, propondrá que se recurra a otro compañero competente en la materia.

b) Si un médico observara que por razón de edad, enfermedad y otras causas, se deteriora su capacidad de juicio o su habilidad técnica, deberá pedir inmediatamente consejo a algún compañero de su absoluta confianza para que le ayude a decidir si debe suspender o modificar temporal o definitivamente su actividad profesional.

c) Si el médico no fuera consciente de tales deficiencias y éstas fueran advertidas por otro compañero, éste está obligado a comunicárselo y, en caso necesario, lo pondrá en conocimiento del Colegio de Médicos, de forma objetiva y con la debida discreción. No supone esta actuación faltar al deber de confraternidad, porque el bien de los pacientes ha de ser siempre prioritario".

- El médico de Atención Primaria o de Salud Mental: Es posible que ante el malestar percibido, el propio trabajador consulte en el Sistema de Salud (en Atención Primaria o Especializada). En ambos casos, disponen de los recursos clínicos de actuación empleados en población general ante un caso de trastorno mental grave, que son de igual manera aplicables al personal sanitario enfermo. Esto es especialmente importante ante la evidencia de una alteración grave del comportamiento que suponga un riesgo para sí mismos o para terceros. Es preceptivo, además, ponerlo en conocimiento al Coordinador del Centro o superior jerárquico, así como al centro de trabajo del paciente (Servicio de Prevención de Riesgos Laborales).

- Colegio Profesional: Cuando este organismo tenga conocimiento de un profesional con un posible trastorno mental grave que suponga riesgo para sí mismo y/o para terceros, se facilitará su evaluación psiquiátrica y velará, según sus propios protocolos de actuación, por la responsabilidad deontológica del profesional sanitario.

- Compañeros/as de trabajo y Superiores jerárquicos: Un compañerismo mal entendido puede llevar a la sobreprotección, ocultación o minimización del problema, facilitando con ello su cronificación e incremento de la gravedad. En el caso de los médicos internos residentes, como señala el PAIME 28 “...entre la estigmatización y la inbibición, el tutor y las personas que supervisan más directamente al residente deben encontrar la situación más conveniente". Se 
sugiere que ante la sospecha o detección de un trastorno mental grave con posible riesgo para sí mismo o para terceros se proceda de la siguiente manera:

- Comunicación verbal con el propio afectado sugiriendo la necesidad de consulta profesional en su S.P.R.L y/o con el superior inmediato del trabajador afectado.

- El Superior le sugerirá verbalmente la posibilidad de consulta con el S.P.R.L.

- Si accede: Ver apartado referido al Servicio de Prevención de Riesgos Laborales (S.P.R.L.)

- Si no accede: Elaboración de un escrito por parte de compañeros y superior, en el que de forma esquemática, fechada y objetiva se señalen conductas disruptivas observadas, conflictos en el lugar de trabajo, sintomatología clínica observada y otros datos de interés. Este escrito se hará llegar al S.P.R.L.

- Servicio de Prevención de Riesgos Laborales (S.P.R.L.): A lo largo del ejercicio profesional en el ámbito sanitario son diversos los momentos en los que cobra especial relevancia la vigilancia de la salud en relación con la capacidad profesional (la incorporación al puesto, cambios sustanciales en el puesto de trabajo, cambios sustanciales en el comportamiento del profesional sanitario, etc.) y son los S.P.R.L. las entidades implicadas en dicha valoración.

En lo relativo a la Ley de Prevención de Riesgos Laborales (Ley 31/1995 y Reglamento 39/1997), según la norma, el reconocimiento es voluntario (es preciso el consentimiento) pero se exceptúan "los supuestos en que la realización de los reconocimientos sea imprescindible para evaluar los efectos de las condiciones de trabajo sobre la salud de los trabajadores, o para verificar si el estado de salud del trabajador puede constituir un peligro para el mismo, para los demás trabajadores o para otras personas relacionadas con la empresa o cuando así esté establecido en una disposición legal en relación con la protección de riesgos específicos y actividades de especial peligrosidad" (artículo 22). Además, será necesario prestar protección a los trabajadores especialmente sensibles a determinados riesgos (art. 25, Ley 31/1995): se "garantizará de manera específica la protección de los trabajadores que, por sus propias características personales o estado biológico conocido, incluidos aquellos que tengan reconocida la situación de discapacidad física, psíquica o sensorial, sean especialmente sensibles a los riesgos derivados del trabajo". Se sugiere para ello:

- Detección o sospecha de trastorno mental grave con riesgo para sí mismo o para terceros durante la vigilancia de la salud en evaluación inicial, en seguimiento o ante petición propia del trabajador. El diagnostico psiquiátrico se realiza mediante la entrevista clínica y de las pruebas clínicas necesarias, habitualmente por un médico psiquiatra.

- Detección o sospecha de trastorno mental grave con riesgo para sí mismo o para terceros mediante escrito de comunicación por parte de compañeros y superior del trabajador: Citación al trabajador para vigilancia de la salud.

- Si acude: Proceder como en apartado anterior.

- Si no acude: Comunicar a Dirección Médica por escrito.

- Dirección/Gerencia: La responsabilidad profesional es una cadena que empieza en el propio sanitario e ineludiblemente continúa en la responsabilidad profesional corporativa, institucional o administrativa. El Director Gerente, como máxima autoridad de la institución sanitaria, es el responsable último del correcto funcionamiento asistencial de la misma. Cuando a través del S.P.R.L. (o por otra vía), tenga conocimiento de la existencia de un trabajador con trastorno mental grave, con riesgo para sí mismo o para terceros, facilitará en la medida de lo posible la exigencia de valoración clínica por un especialista en salud mental. Si no existe conciencia de enfermedad, las medidas preventivas se podrán tomar en función de diferentes vías, no excluyentes entre sí:

- Ley de Prevención de Riesgos Laborales: Por el posible riesgo para sí mismo y/o para terceros en el lugar de trabajo. 
- Inspección Sanitaria: Si existe riesgo de daño.

- Colegio Profesional: Para la prevención de mala praxis y evaluación deontológica del caso.

- Urgencias: A través del SUMMA 112 y comunicación al Juez, si se considera necesaria una intervención clínica no voluntaria.

Existe apoyo legal para todas esas medidas. Por ejemplo, la embriaguez o toxicomanía habitual puede ser causa de despido (art. 54 Estatuto de los Trabajadores, Ley 8/1980 de 10 de marzo) si se trata de una conducta reiterativa y repercute negativamente en el trabajo (quejas de terceros, repercusiones negativas para la organización, ausencias al trabajo, etc.) y es probada objetivamente. Además, según la aplicación del Estatuto Marco [Ley 55/2003, de 16 de diciembre, del Estatuto Marco del Personal Estatutario de los Servicios de Salud, artículos 19.2.m); 21.f); 19.3.m); 27; 72.2. f), g) y u) y artículo 75], entre los deberes del personal está el de cumplir las normas relativas a la seguridad y salud en el trabajo, así como las disposiciones adoptadas en el centro sanitario en relación con esta materia; siendo una causa de extinción de la condición de personal estatutario fijo la incapacidad permanente cuando sea declarada en sus grados, entre otros, de incapacidad permanente total para la profesión habitual; constituyendo falta muy grave el notorio incumplimiento de sus funciones o de las normas reguladoras del funcionamiento de los servicios, así como la desobediencia notoria y manifiesta a las órdenes o instrucciones de un superior directo, mediato o inmediato, emitidas por éste en el ejercicio de sus funciones y la negativa expresa a hacer uso de los medios de protección disponibles y seguir las recomendaciones establecidas para la prevención de riesgos laborales, así como la negligencia en el cumplimiento de las disposiciones sobre seguridad y salud en el trabajo por parte de quien tuviera la responsabilidad de hacerlas cumplir o de establecer los medios adecuados de protección.

Además, como medida cautelar, y durante la tramitación de un expediente disciplinario por falta grave o muy grave o de un expediente judicial, podrá acordarse mediante resolución motivada la suspensión provisional de funciones del interesado, en los términos establecidos en dicho Estatuto.

\section{Diagnóstico e intervención en el caso del personal con probable trastorno mental grave y posible riesgo para sí mismo o para terceros}

Cuando, según los criterios mencionados, se identifique a un trabajador como posible trastorno mental grave con riesgo para sí mismo o para terceras personas se agilizará, en la medida de lo posible, la primera visita a un servicio especializado en salud mental para la evaluación del caso. En él se analizará la necesidad de un tratamiento asistencial psicofarmacológico y se recomienda valorar los siguientes aspectos fundamentales:

- Valoración del grado de discapacidad mental y necesidad de incapacidad temporal (en un primer momento) y/o permanente (si se considera oportuno a lo largo del proceso asistencial).

- Conveniencia de intervención médica y psiquiátrica hospitalaria urgente (voluntaria o involuntaria) en su Servicio de Urgencias correspondiente.

- Comunicación con la familia del profesional sanitario con posible trastorno mental grave.

- En caso de médicos internos residentes, comunicación con su tutor de residentes.

- Comunicación con el S.P.R.L. al que esté adscrito el profesional sanitario, a fin de controlar los posibles riesgos laborales que puedan verse incrementados por sus condiciones de salud y valoración del riesgo para sí mismo y para terceros de forma extensible al lugar de trabajo. Es función de este servicio la valoración sobre la aptitud o no del paciente en su puesto de trabajo, su posible consideración como trabajador especialmente sensible y la valoración del riesgo profesional. 
- Si el S.P.R.L. lo considera oportuno tras el proceso de vigilancia de la salud y evaluación de aptitud profesional, comunicará el riesgo a Gerencia. Si el enfermo no acepta el criterio de no aptitud para el trabajo, pero se estima riesgo para terceros, se recomienda notificar esta contingencia a la Inspección Sanitaria (que puede aplicar igualmente la baja laboral por motivos médicos según normativa legal vigente), y por él la Gerencia Sanitaria tiene la responsabilidad sobre el funcionamiento asistencial de su entidad sanitaria, por lo que, si lo considera oportuno, comunicará el riesgo para terceros al juez o fiscalía de incapacidades, así como la oportunidad de valorar la responsabilidad deontológica y posible riesgo de mala praxis por parte del Colegio Profesional del trabajador enfermo.

\section{Otras actuaciones:}

A lo largo de todo el proceso de detección, evaluación e intervención, pueden ser necesarias otro tipo de actuaciones no mencionadas anteriormente:

1. Aspectos relativos a la incapacidad laboral: En los casos de trastorno mental grave, se valorará la necesidad de gestionar una incapacidad profesional.

- Temporal o transitoria (mediante informe clínico dirigido a su médico de Atención Primaria): cuando se prevea una recuperación en un período de tiempo determinado. Se definirá por:

- Incapacidad para el trabajo por causa sanitaria.

- Carácter temporal en función del pronóstico.

- Permanente (mediante informe clínico y psicosocial dirigido a su Equipo de Valoración de Incapacidades): cuando se presente una alteración de la capacidad laboral previsiblemente definitiva. A su vez, ésta puede ser:

- Invalidez Permanente Parcial: Cuando se ocasiona una disminución no inferior al 33\% de su rendimiento normal para su profesión habitual.

- Invalidez Permanente Total: Inhabilita al trabajador para todas las tareas de su profesión habitual.

- Invalidez Permanente Absoluta: Inhabilita para toda profesión u oficio.

- Gran Invalidez: Cuando sea necesaria la asistencia de otras personas para realizar los actos esenciales de la vida.

2. Aspectos relativos a la incapacitación (civil): Es necesario recordar que:

- Nadie puede ser declarado incapaz sino por sentencia judicial en virtud de las causas establecidas en la Ley.

- Son causas de incapacitación las enfermedades o deficiencias persistentes de carácter físico o psíquico que impidan a la persona gobernarse por sí misma.

- Existen diferentes grados de incapacidad. El juez, tras informe del médico forense, valorará el tipo de incapacidad.

Cuando en la valoración y tratamiento del paciente con trastorno mental grave se presente dicha situación, se pondrá en conocimiento del propio paciente y/o familiares del mismo para la gestión del proceso de incapacitación:

- La declaración de incapacidad la promoverán el presunto incapaz, el cónyuge o quien se encuentre en una situación de hecho asimilable (descendientes, ascendientes, hermanos).

- El Ministerio Fiscal deberá promover la incapacitación si las personas mencionadas en el apartado anterior no existieran o no la hubieran solicitado.

- Según la legislación vigente, cualquier persona está facultada para poner en conocimiento del Ministerio Fiscal los hechos que puedan ser determinantes de la incapacitación. Las autoridades y funcionarios públicos que, por razón de sus cargos, conocieran la existencia de posible causa de incapacitación en una persona, deberán ponerlo en conocimiento del Ministerio Fiscal. 


\section{Tratamiento Ambulatorio No voluntario:}

Cuando exista falta de capacidad de decisión del paciente y así lo requiera la salud del enfermo, por razón del trastorno psíquico o por necesidad de un periodo de observación para el diagnóstico, se podrá promover la gestión del tratamiento psiquiátrico ambulatorio involuntario:

- Podrán promover este expediente el cónyuge del paciente o quien se encuentre en una situación de hecho asimilable, descendientes, ascendientes, hermanos, tutores, así como el facultativo que atienda al paciente.

- La solicitud de autorización de tratamiento no voluntario se realizará mediante propuesta razonada sobre la situación de incapacidad del paciente, el tratamiento al que está sometido y la situación de incumplimiento del mismo.

- El tratamiento propuesto podrá cesar por prescripción facultativa, comunicándose esta medida al Juzgado de Primera Instancia.

- En el caso de autorización de un periodo de observación para diagnóstico, el informe deberá remitirse al tribunal en el plazo máximo de dos meses, tras los cuales se podrá solicitar, si procede, la autorización de internamiento o tratamiento no voluntario.

4. Internamiento Involuntario.

El Consejo de Europa (2004) establece una recomendación (cap. III, art. 17) en el que expone los siguientes criterios normativos para la hospitalización psiquiátrica involuntaria:

- La persona tiene un trastorno mental grave.

- Existe riesgo significativo de daño para su salud o la de otros.

- El ingreso tiene objetivo terapéutico.

- No hay otro medio menos restrictivo de ofrecer la atención apropiada.

- Se tendrá en consideración la opinión de la persona afectada, convenientemente informada.

Cuando durante el proceso de valoración diagnóstica del trastorno mental grave se detecte la necesidad de internamiento en una unidad hospitalaria de forma involuntaria se seguirá el artículo 763 de la Ley de Enjuiciamiento Civil sobre internamiento involuntario por razón de trastorno psíquico:

- El internamiento, por razón de trastorno psíquico, de una persona que no esté en condiciones de decidirlo por sí, aunque esté sometida a la patria potestad o a tutela, requerirá autorización judicial, que será recabada del tribunal del lugar donde resida la persona afectada por el internamiento.

- La autorización será previa a dicho internamiento, salvo que razones de urgencia hicieren necesaria la inmediata adopción de la medida. En este caso, el responsable del centro en el que se hubiere producido el internamiento deberá dar cuenta de éste al tribunal competente lo antes posible (plazo de 24 horas).

- En los casos de internamiento urgentes, la competencia para la ratificación de la medida corresponderá al tribunal del lugar en que radique el centro donde se haya producido el internamiento.

\section{Información al paciente con posible riesgo para sí mismo y/o para terceros y plan terapéutico}

Todos los pacientes que, a lo largo del proceso de evaluación y/o tratamiento, se califiquen como "trastorno mental grave y riesgo para sí mismo o para terceras personas" serán informados de tal condición.

Si el paciente acepta dicha condición:

- Se recomienda que firme un contrato terapéutico comprometiéndose a seguir el plan terapéutico propuesto, previa valoración de su capacidad ${ }^{7}$. 
- En el caso de pacientes con problemas adictivos, dicho plan comportará la realización de un tratamiento de desintoxicación, deshabituación y rehabilitación, con el seguimiento posterior y controles toxicológicos necesarios según criterio clínico.

Si el paciente no acepta dicha condición o existe incumplimiento del plan terapéutico:

- Se considerará la necesidad de gestionar el tratamiento novoluntario o el internamiento involuntario mediante solicitud judicial.

- Se informará de forma inmediata a su Servicio de Prevención de Riesgos Laborales, a fin de controlar los posibles riesgos laborales que puedan verse incrementados por sus condiciones de salud y valoración del riesgo para si mismo y para terceros de forma extensible al lugar de trabajo (será función de este servicio la valoración sobre la aptitud o no del paciente en su puesto de trabajo, su posible consideración como trabajador especialmente sensible y la valoración del riesgo profesional).

- Si el S.P.R.L. lo considera oportuno tras el proceso de vigilancia de la salud y evaluación de aptitud profesional, comunicará el riesgo a Gerencia.

- La Gerencia Sanitaria tiene la responsabilidad sobre el funcionamiento asistencial de su entidad sanitaria, por lo que, si lo considera oportuno, comunicará el riesgo para terceros al juez y la oportunidad de valorar la responsabilidad deontológica y posible riesgo de mala praxis al Colegio Profesional del sanitario enfermo.

\section{Seguimiento}

Los pacientes atendidos podrán ser derivados a las unidades más convenientes del Servicio Madrileño de Salud y/o a los Servicios Asistenciales con los que exista un convenio de colaboración y garantizar así la mejor calidad asistencial, confidencialidad, seguridad y seguimiento compartido del profesional enfermo.

\section{CONCLUSIONES}

El constructo "trastorno mental grave" incluye un conjunto de diferentes entidades nosológicas, que se presentan en una persona de forma continua o episódica-recurrente durante unos dos años (de media) y que cumplen unos determinados criterios diagnósticos de gravedad clínica y de evolución crónica; de forma que, para atenderlos, se debe disponer de unos recursos asistenciales adecuados.

Actualmente, hay un amplio acuerdo entre profesionales y gestores acerca de que debe priorizarse la atención en los Servicios de Salud Mental a las personas que padecen un trastorno mental grave, ya que son las que suelen tener una mayor discapacidad derivada del padecimiento del mismo. Por otro lado, es necesario disponer de instrumentos que mejoren el reconocimiento de los casos más graves y ayuden a la toma de decisiones en su derivación ${ }^{29,30}$.

Se debe separar con claridad la asistencia clínica a un profesional sanitario enfermo de la evaluación pericial sobre su capacidad de ejercicio profesional. Esta última evaluación deberá ser llevada a cabo por un equipo de profesionales independiente, sin posibles conflictos de intereses administrativos y/o clínicos ${ }^{31}$. La competencia profesional del personal sanitario implica aprendizaje y formación continuada, tanto de competencias científico-técnicas como psicosociales; implica la posibilidad de establecer relaciones éticamente adecuadas con los pacientes (donde exista el compromiso de confidencialidad, la búsqueda de la adherencia terapéutica, de las responsabilidades profesionales y la calidad asistencial). Estas obligaciones del personal asistencial requieren de procesos de autorregulación, de identificación, notificación y, sobre todo, ayuda, a los profesionales que no cumplan los estándares de competencia profesional por motivos relacionados con su salud mental. 
Como ya se ha señalado a lo largo de este trabajo, el personal sanitario puede resultar no capacitado para el desarrollo de sus funciones asistenciales debido a la existencia de un trastorno mental grave y/o adictivo por su afección de capacidades cognitivas, afectivas y conductuales. La responsabilidad ética, deontológica y legal de comunicar esta situación es universal y debe hacerse llegar a la autoridad médica competente. Desde la doctrina bioética moderna se espera que todos los profesionales sanitarios participemos de forma solidaria y responsable en un proceso autorregulador eficaz, lo que supone la rectificación y la sanción de los profesionales que no sean capaces de cumplir con los estándares de calidad y de competencia profesional esperables.

La complejidad de las relaciones que derivan de la actuación en equipo (unidad básica según la actual Ley de Ordenación de las Profesiones Sanitarias, de 2003), ha llevado a nuestra jurisprudencia (Tribunal Supremo) a delimitar las diferentes responsabilidades a través de lo que se conoce como "principio de la confianza". Consiste en síntesis en que el médico que actúa de forma correcta puede confiar en que los demás miembros del equipo también lo harán de igual forma, salvo que se den circunstancias especiales que hagan pensar en lo contrario. Ahora bien, el principio de la confianza quiebra, esto es, ya no se puede confiar, como puede ocurrir en caso de trastorno mental grave, y hay que hacer algo en defensa del paciente para no incurrir en responsabilidad. Cada uno es, pues, responsable de sus actos, pero está obligado a hacer algo cuando se rompe el principio de la confianza, pues en otro caso todos los que sean conscientes de la situación serán responsables del daño causado al paciente.

Este artículo es resultado del esfuerzo colectivo de esta Unidad, y pretende dar una respuesta a las demandas que plantea el padecimiento de una enfermedad mental por parte de los profesionales sanitarios. Se trata de casos complejos y difíciles de resolver por sus características y circunstancias, de manera que solo la experiencia que se adquiera con la puesta en marcha del presente protocolo será lo que nos indique su valor y posibilidades de mejora.

\section{BIBLIOGRAFÍA}

1. Jaspers, K. Psicopatología General. Primera edición en 1913. Ed. Beta. Buenos Aires, 1963.

2. Kendell, R. Jablensky, A. Distinguishing between the validity and utility of psychiatric diagnosis. American Journal of Psychiatry 2003, 160 (1):4-12.

3. Epstein, RM; Hundert, EM. Defining ans assessing professional competente. JAMA 2002, Jan 9; 287,2: $226-235$.

4. Epstein, RM et al. Comprenhensive assessemante of professional competente: the Rochester experiement. Teach learn med 2004; 16 (2): 186-196IR.

5. Consejo Internacional de Enfermeras. Código Deontológico para la Profesión de Enfermería 2006.

6. Nogales, A.; García-Seoane, J.; Calvo, E., y Grupo de Trabajo Para la Definición de Competencias. Competencias para el Grado de Medicina de la Universidad Complutense de Madrid. Documento Base. Unión Editorial: Madrid, 2008.

7. Boada, M. y Robles, A. (Eds.). Documento Sitges 2009. Editorial Glosa, S.L.: Barcelona, 2008.

8. Gold, LH. Shuman, DW. Evaluating Mental Health Disability in the Workplace. DSpringer. Londres, 2009.

9. Meyerson, A. Fine, Th. Psychiatric Disability. Clinical, Legal and Administrative Dimensions. American Psychiatric Press. Washington, 2005.

10. Center of Mental Health Services (USA). Substance Abuse and Mental Health Service Administration: Final Notice Establishing Definitions for Children with a Serious Emotional Disturbance and Adults with a Serious Mental Illness. Federal Register, 1993; 58 (96): 29422-29425.

11. Thornicroft, G., y Tansella, M. La Matriz de la Salud Mental. Manual para la mejora de servicios. Fundación Española de Psiquiatría y Salud Mental. Madrid: Editorial Triacastela, 2005.

12. Slade, M.; Powell, G. y Strahdee, G. Current approaches to identifying the severely mentally ill. Social Psychiatry Epidemiology 1997, 32: 177-184. 
13. Ruggieri, M; Leese, M; Thornicroft, G. y cols. Definition and prevalence of severe and persistent mental illness. British Jounal of Psychiatry, 2000, 177: 149-155

14. Pichot P. Evaluación de los trastornos psiquiátricos graves. En: Diagnóstico y evaluación de los trastornos psiquiátricos graves. Aula Médica. Madrid, 2005.

15. Opler, L.A.; Ramírez, P.M. y Mougios, V.M. Medida de resultados en las enfermedades mentales graves. En: Waguih William IsHak, Tal Burt, Lloyd I. Sederer. Medida de Resultados en Psiquiatría. Una revisión exhaustiva. Ars Medica: Barcelona, 2004.

16. Schinnar, AP; Rothbar, AB; Kanter, R. y cols. An empirical literature review of definitions of severe and persistent mental illness. American Journal Psychiatry 1990, 147: 1602-1608.

17. Addington, J; Addington, D. Attentional vulnerability indicators in schizophrenia and bipolar disorders. Schizophrenia Research 1997, 23: 197-204.

18. Tirapu, Ríos y Maestú. Manual de Neuropsicología. Viguera. Barcelona, 2008.

19. Ramírez, M; Enjuto, S; Aldama, A y col. Neuropsicología de los trastornos esquizoafectivos. En: Ciclo vital y trastornos psiquiátricos graves. Ed. Pichot. Aula Médica. Madrid. 2002.

20. Kessler, RC; Berglund, P; Demler, P y col. Lifetime prevalence and age-of-onset distributions of DSM-IV in the National Comorbity Survey Replication. Archives of General Psychiatry 2005, 62: 593-602.

21. David, AS; Buchnan, A; Reed, A; Almeida, O. Escala de Evaluación del Insight (SAI9. The assessment of insinght in psicosis. Bristish Journal Psychiatry 1992; 161:599-602.

22. Bulbena Vilarrasa, A; Zúñiga Lagares, A; Martín Carrasco, M; Ballesteros Rodríguez, J. Escala de Gravedad de la Enfermedad Psiquiátrica para pacientes ambulatorios (Gep-P). Gravedad clínica en psiquiatría. Cap. 5 en: Medición clínica en Psiquiatría y Psicología. Antonio Bulbena Vilarrasa, Germán E. Barrios, Pedro Fernández de Larrinoa Palacios. MASSON. Barcelona, 2000.

23. Grisso, T; Appelbaum, PS; Assessing Competente to Consent to Treatment. Oxford University Press. New Cork. 1998. Instrumento Mac Arthur para la Evaluación de la capacidad sobre el tratamiento (Mac Cat-T).

24. Sims, A. Síntomas mentales. Tricastela. Madrid. 2008.

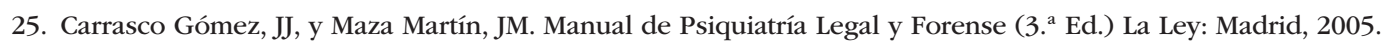

26. Bravo Ortiz, MF. Rehabilitación psiquiátrica y orientación a la familia. En Manual de Psiquiatría. Tomás Palomo y Miguel Ángel Jiménez Arriero (Eds). Enc. Life Publicidad, S.A., Editores. Capítulo 59:869-885. Madrid, 2009.

27. Comisión Central de Deontología, Derecho Médico y Visado. Código de Deontología Médica. Consejo General de los Colegios Oficiales Médicos. Madrid, 1985.

28. PAIME. La salud del MIR. Guía para tutores y profesionales de los centros sanitarios docentes. Fundación Galatea. Barcelona, 2008.

29. Moré, MA, Coordinador del Grupo de Trabajo Asociación Madrileña de Salud Mental. Prestaciones básicas y estándares de calidad en los servicios de salud mental. Cuaderno Técnico n. ${ }^{\circ}$ 1. AMSM: Madrid, 1998.

30. Moré, M.A.; Jiménez, M.A.; Muñóz, P.E.; Muñoz, A., y Zufía, J. Estudio preliminar dirigido a la construcción de un cuestionario de derivación de la atención primaria a los servicios de salud mental. Actas Españolas de Psiquiatría 2008; 36(4); 210-217.

31. Sadock, B.J. y Sadock, V.A. Sinopsis de Psiquiatría. Waverly Hispánica, S.A. Barcelona, 2004.

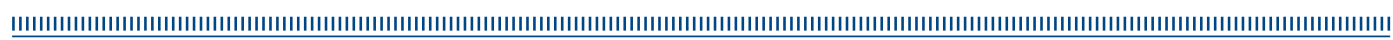

